\title{
Geheime Verhandlungen: Neuer EBM soll 2019 kommen
}

— KBV und der GKV-Spitzenverband haben im Bewertungsausschuss am 29. März 2017 beschlossen, dass der Zeitplan zur Weiterentwicklung des EBM angepasst wird. Der Öffentlichkeit war von einem solchen Plan bisher noch überhaupt nichts bekannt. Umso erstaunlicher ist es, dass der neue EBM

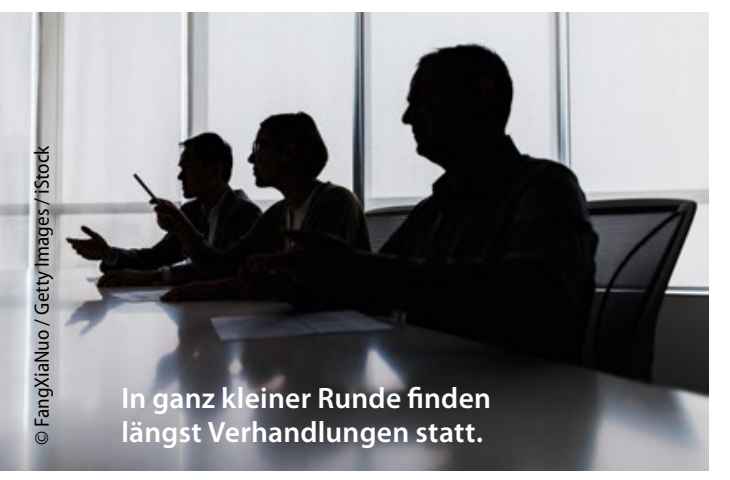

nach den bisherigen Vorstellungen bereits zum 1. Juli 2017 in Kraft treten sollte. Nun soll bis zum 30. September 2018 beraten werden, was im haus- und im fachärztlichen Bereich geändert werden soll. Als Start für die neuen Regeln ist der 1. Januar 2019 vorgesehen.

\section{MMW-KOMMENTAR}

Offensichtlich hat die KBV ihre Lehren aus den endlosen Diskussionen um eine Novellierung der GOÄ gezogen. Die parallel laufenden EBM-Beratungen sind deshalb ziemlich geheim gehalten worden. Details sind wohl nur absoluten Insidern bekannt. Die Beratungen fanden bisher offenbar in ganz kleinem Rahmen statt. Ob dabei breiter Sachverstand eingeflossen ist, darf bezweifelt werden. Angesichts dieser Sachlage dürfte ein Widerstand der Berufs- und Fachverbände nun

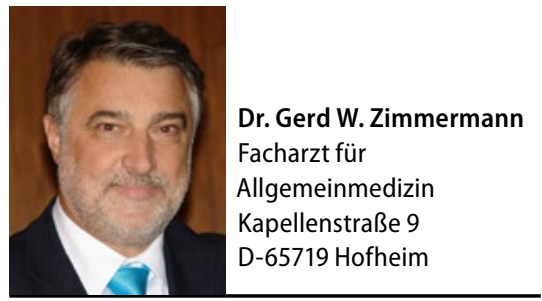

kaum zu vermeiden sein - auch das kennen wir noch von der GOÄ-Novellierung. Bekanntlich konnte dort erst im Rahmen eines Sonder-Ärztetags eine breitere Basis für die Beratungen erreicht werden. Um eine Sonder-Vertreterversammlung zur EBM-Reform dürfte die KBV deshalb auch nicht herumkommen.

Bisher ist nur bekannt, dass es zu einer Überprüfung des EBM sowie zu Änderungen im Standardbewertungssystem (STABS) und parallel zu einer fristgerechten Umsetzung gesetzlicher Aufträge kommen soll. Die EBMAnpassungen sollen weiterhin auf einer Datengrundlage erfolgen, die das aktuelle Versorgungsgeschehen möglichst gut abbildet. Hier sollen die Ergebnisse der neuesten Kostenstrukturerhebungen in Praxen einflieBen, die das Statistische Bundesamt im Herbst vorlegen will.

\section{Klinik-Entlassmanagement startet später}

_ Patienten haben ab dem 1. Juli 2017 gegenüber Krankenhäusern Anspruch auf ein Entlassmanagement inklusive Entlassgespräch und -brief (siehe MMW 21-22/2016 und 2/2017). Leistungen wie häusliche Krankenpflege, Hilfsmittel oder Maßnahmen zur Rehabilitation, die vorher bei der Kasse beantragt und genehmigt werden müssen, sollen bereits vor der Entlassung durch die Klinik verordnet werden. Gleiches gilt für die Verordnung von Arznei-, Verband-, Heilund Hilfsmitteln sowie Soziotherapie und für die Feststellung der Arbeitsunfähigkeit. Außerdem erhält der Patient einen Medikationsplan, der wie ein in der Arztpraxis erstellter aussieht.
Die Krankenhäuser müssen die weiterbehandelnden Ärzte so früh wie möglich einbeziehen und ihnen einen generellen Ansprechpartner nennen.

\section{MMW-KOMMENTAR}

Die Einführung eines Krankenhaus-Entlassmanagements setzt voraus, dass auch Krankenhäuser eine Betriebsstättennummer und Krankenhausärzte eine Arztnummer erhalten. Nach anfänglichen Widerständen der Krankenhäuser haben sich nun der GKV-Spitzenverband, die KBV und die Deutsche Krankenhausgesellschaft (DKG) darauf verständigt, eine Gesetzesänderung zur Einführung einer Krankenhausarztnummer zu unterstützen. Das Entlassmanagement star- tet deshalb erst zum 1. Oktober 2017 - und nicht schon zum 1. Juli, wie ursprünglich vorgesehen.

Ab Januar 2019 wird dann die Krankenhausarztnummer eingeführt. Bis dahin verwenden Krankenhausärzte übergangsweise ein Arztpseudonym zur Kennzeichnung von Verordnungen. Diese Verfahrensänderung hat allerdings keine Auswirkungen auf die Entlastung der Praxen, die mit der Einführung des Entlassmanagements beabsichtigt ist. Es ist deshalb ratsam, den Patienten oder dessen Angehörige ab dem 1. Oktober 2017 nach einer stationären Einweisung auf das Recht auf ein Entlassmanagement hinzuweisen, damit das Krankenhaus dies auch umsetzt und die Praxis entlastet. 